\title{
Arithmetic Clifford's theorem for Hermitian vector bundles
}

\author{
by \\ Tohru NAKAShima (Tokyo)
}

1. Introduction. Let $K$ be a number field and let $\mathcal{O}_{K}$ denote its ring of integers. A Hermitian vector bundle $\bar{E}$ on an arithmetic curve $\operatorname{Spec} \mathcal{O}_{K}$ is a projective $\mathcal{O}_{K}$-module $E$ equipped with a Hermitian metric $E \otimes_{v} \mathbb{C}$ for each infinite place $v: K \hookrightarrow \mathbb{C}$. We expect that Hermitian bundles have properties similar to those of vector bundles on an algebraic curve defined over a field.

Recently the notion of size $h^{0}(\bar{L})$ has been introduced for a Hermitian line bundle $\bar{L}$ on an arithmetic curve. This invariant may be considered as an arithmetic analogue of the dimension of the space of global sections. For example, a Riemann-Roch type theorem holds for them ([GS]).

In $[G]$ Groenewegen has proved an arithmetic analogue of Clifford's theorem by means of the size function. The purpose of this note is to generalize the result to semistable Hermitian vector bundles. We notice that this is also an arithmetic analogue of a theorem obtained in [BGN] in the geometric case. We also consider an example of a semistable Hermitian bundle which comes from arithmetic abelian schemes ([B]).

The author thanks the referee for correcting mistakes in the original manuscript and for valuable suggestions.

2. Statement of the result. Let $K$ be a number field and let $S^{\infty}$ denote the set of infinite places of $K$. The scheme $S=\operatorname{Spec} \mathcal{O}_{K}$ is said to be an arithmetic curve. If we denote by $r_{1}$ and $r_{2}$ the number of real resp. complex embeddings of $K$, then $n=[K: \mathbb{Q}]=r_{1}+2 r_{2}$.

Let $\bar{E}$ be a Hermitian vector bundle of rank $r$ on $S$. In other words, $E$ is a projective $\mathcal{O}_{K}$-module of rank $r$ equipped with Hermitian metrics \|\|$_{v}$ on the complex vector spaces $E_{v}=E \otimes_{v} \mathbb{C}$ associated with the embeddings

2000 Mathematics Subject Classification: 11R04, 14G40.

Key words and phrases: arithmetic curves, Hermitian bundles, Clifford's theorem. 
$v \in S^{\infty}$. Let

$$
E_{\mathbb{C}} \cong \bigoplus_{v \in S^{\infty}} E_{v}
$$

Let $E \otimes_{\mathbb{Z}} \mathbb{R}$ denote the real subspace of $E_{\mathbb{C}}$ fixed under complex conjugation. We equip $E_{\mathbb{R}}$ with the norm which is the restriction of the norm on $E_{\mathbb{C}}$ which is defined for $x \in E_{\mathbb{C}}$ as follows:

$$
\|x\|^{2}=\sum_{v \in S^{\infty}}\|x\|_{v}^{2} .
$$

We consider $E$ as a lattice of rank $r n$ in $E_{\mathbb{R}}$.

The arithmetic degree of a Hermitian line bundle $\bar{L}$ is defined by

$$
\widehat{\operatorname{deg}} \bar{L}=\log \sharp\left(L / \mathcal{O}_{K} s\right)-\sum_{v \in S^{\infty}} \log \|s\|_{v}
$$

where $s$ is a nonzero element of $L$. For a general Hermitian bundle $\bar{E}$, we define $\widehat{\operatorname{deg}} \bar{E}$ by

$$
\widehat{\operatorname{deg}} \bar{E}=\widehat{\operatorname{deg}} \operatorname{det} \bar{E} \text {. }
$$

We have the equality

$$
\widehat{\operatorname{deg}} \bar{E}=-\log \operatorname{covol} \bar{E}+\frac{r \log \left|\Delta_{K}\right|}{2}
$$

where we denote by $\operatorname{covol} \bar{E}$ the covolume of $\bar{E}$ and by $\Delta_{K}$ the discriminant of $K$. We define the norm of $\bar{E}$ by

$$
N(\bar{E})=e^{\widehat{\operatorname{deg}} \bar{E}} .
$$

The $\mathcal{O}_{K}$-module

$$
\omega_{S}=\operatorname{Hom}_{\mathbb{Z}}\left(\mathcal{O}_{K}, \mathbb{Z}\right)
$$

is locally free of rank one. We equip $\omega_{S}$ with the Hermitian metric which is defined, for each $v \in S^{\infty}$, by $\|\operatorname{Tr}\|_{v}=1$ for the trace map $\operatorname{Tr} \in \omega_{S}$. Let $\bar{\omega}_{S}$ denote the resulting Hermitian line bundle. Then we have

$$
\widehat{\operatorname{deg}} \bar{\omega}_{S}=\log \left|\Delta_{K}\right| \text {. }
$$

For a Hermitian vector bundle $\bar{E}$, we denote by $\bar{E}^{\vee}=\operatorname{Hom}_{\mathcal{O}_{K}}\left(E, \mathcal{O}_{K}\right)$ the dual vector bundle equipped with the dual metric. We let

$$
\bar{E}^{*}=\bar{E}^{\vee} \otimes \bar{\omega}_{S}
$$

denote the Hermitian bundle equipped with the tensor product of the dual metric and the metric on $\omega_{S}$ defined above. Its degree is given by

$$
\widehat{\operatorname{deg}} \bar{E}^{*}=-\widehat{\operatorname{deg}} \bar{E}+r \widehat{\operatorname{deg}} \bar{\omega}_{S} .
$$

Following [GS], we define the effectivity of $\bar{E}$ to be the number

$$
k^{0}(\bar{E})=\sum_{x \in E} e^{-\pi\|x\|^{2}}
$$


Then the size of $\bar{E}$ is defined as follows:

$$
h^{0}(\bar{E})=\log k^{0}(\bar{E}) .
$$

$h^{0}(\bar{E})$ may be considered as an arithmetic analogue of the dimension of the space of global sections in the geometric case. For example, as a consequence of the Poisson summation formula we have the following result, which is stated in [GS, 9] in a different form.

Proposition 2.1 (Riemann-Roch). Let $\bar{E}$ be a Hermitian vector bundle of rank $r$ on $S$. Then

$$
h^{0}(\bar{E})-\widehat{\operatorname{deg}} \bar{E} / 2=h^{0}\left(\bar{E}^{*}\right)-\widehat{\operatorname{deg}} \bar{E}^{*} / 2 .
$$

For a Hermitian bundle $\bar{E}$, we define its slope $\widehat{\mu}(\bar{E})$ by

$$
\widehat{\mu}(\bar{E})=\widehat{\operatorname{deg}} \bar{E} / \mathrm{rk} E .
$$

$\bar{E}$ is said to be semistable if $\widehat{\mu}(\bar{F}) \leq \widehat{\mu}(\bar{E})$ for every subbundle $\bar{F}$ of $\bar{E}$ with the induced metric.

The main result of this note is the following

THEOREM 2.2. Let $\bar{E}$ be a semistable Hermitian vector bundle of rank $r$ on $S=\operatorname{Spec} \mathcal{O}_{K}$.

(1) If $\widehat{\operatorname{deg}} \bar{E} \leq 0$, then

$$
h^{0}(\bar{E})<\frac{3^{r n} \pi}{\pi-r \log 3} e^{-\pi e^{-2(\widehat{\operatorname{deg}} \bar{E}) /(r n)} .}
$$

(2) If $\widehat{\operatorname{deg}} \bar{E} \geq 0$, then

$$
h^{0}(\bar{E}) \leq r n\left(\log \omega+\log r+2^{-1} \log n\right)+\widehat{\operatorname{deg}} \bar{E} .
$$

(3) If $0 \leq \widehat{\mu}(\bar{E}) \leq \widehat{\operatorname{deg}} \bar{\omega}_{S}$, then

$$
h^{0}(\bar{E}) \leq r n(\log \omega+\log r+\log n)+\widehat{\operatorname{deg}} \bar{E} / 2 .
$$

We notice that the above theorem has a geometric counterpart. Let $C$ be a smooth projective curve over $\mathbb{C}$ and let $E$ be a semistable vector bundle of rank $r$ on $C$. Let $\mu(E)=\operatorname{deg} E / r$ denote its slope. Then $h^{0}(E)=\operatorname{dim} H^{0}(E)$ has the following properties.

Proposition 2.3. (1) If $\operatorname{deg} E<0$, then $h^{0}(E)=0$.

(2) If $\operatorname{deg} E \geq 0$, then $h^{0}(E) \leq r+\operatorname{deg} E$.

(3) If $0 \leq \mu(E) \leq 2 g-2$, then $h^{0}(E) \leq r+\operatorname{deg} E / 2$.

Proof. (1) is clear from the definition and (3) has been proved in [BGN, Theorem 2.1]. We prove (2) by induction on $r$, following the argument in loc.cit. The case $r=1$ is easy. Assume that the inequality holds for semistable bundles of rank less than $r$. Let $E_{1}$ denote a subbundle of $E$ which has the maximal slope among all proper subbundles. Let $E_{2}=E / E_{1}$. It is 
clear that $E_{1}, E_{2}$ are both semistable. Since we may assume that $h^{0}(E)>0$, we obtain $\mu\left(E_{1}\right) \geq 0$. We also have $\mu\left(E_{1}\right) \geq \mu(E) \geq 0$ by the semistability of $E$. Hence by the induction assumption we obtain $h^{0}(E) \leq h^{0}\left(E_{1}\right)+h^{0}\left(E_{2}\right) \leq \operatorname{rk} E_{1}+\operatorname{deg} E_{1}+\operatorname{rk} E_{2}+\operatorname{deg} E_{2}=r+\operatorname{deg} E$.

3. Proof of Theorem 2.2. For a lattice $\Lambda$, its minimum is defined to be the minimum norm of nonzero elements in $\Lambda$. The following result has been proved by Groenewegen ([G, Proposition 5.4, Corollary 5.7]).

Lemma 3.1. Let $\Lambda$ be a lattice of rank $n$ with minimum $\lambda$ and let $\Lambda^{*}$ denote the dual lattice with minimum $\lambda^{*}$. Let $\omega=k^{0}(\mathbb{Z})$ where $\mathbb{Z}$ is equipped with the trivial metric. For $1 \leq i \leq n$, let $\gamma_{i}$ denote the ith Hermite constant. Then

$$
k^{0}(\Lambda) \leq \omega^{n} \prod_{i=1}^{n} \max \left\{1, \gamma_{i} / \lambda\right\} .
$$

Furthermore, we have either

$$
k^{0}(\Lambda) \leq \omega^{n} \max \{1,1 / \lambda\}^{n / 2} n^{n} \quad \text { or } \quad k^{0}\left(\Lambda^{*}\right) \leq \omega^{n} \max \left\{1,1 / \lambda^{*}\right\}^{n / 2} n^{n} .
$$

Lemma 3.2. If $\bar{E}$ is a semistable Hermitian bundle, then $\bar{E}^{*}$ is also semistable.

Proof. Assume that $\bar{E}$ is semistable and let $\bar{F} \subset \bar{E}^{*}$ be a sub- $\mathcal{O}_{K}$-module with the induced metric. Considering the saturation of $\bar{F}$, we may assume that $\bar{E}^{*} / \bar{F}$ is projective. Then we obtain an injection $\left(\bar{E}^{*} / \bar{F}\right)^{*} \hookrightarrow \bar{E}$. Since $\bar{E}$ is assumed to be semistable, we have $\widehat{\mu}\left(\left(\bar{E}^{*} / \bar{F}\right)^{*}\right) \leq \widehat{\mu}(\bar{E})$, which implies $\widehat{\mu}(\bar{F}) \leq \widehat{\mu}\left(\bar{E}^{*}\right)$ as desired.

Let $\lambda$ (resp. $\left.\lambda^{*}\right)$ denote the minimum of $\bar{E}$ (resp. $\bar{E}^{*}$ ). Since $\bar{E}$ is semistable, so is $\bar{E}^{*}$ by Lemma 3.2. For any nonzero $s \in E$, let $\bar{L}$ denote the Hermitian line bundle which is generated by $s$ in $E$, with the induced metric. Then

$$
\widehat{\mu}(\bar{E}) \geq \widehat{\operatorname{deg}} \bar{L} \geq-\sum_{v \in S^{\infty}} \log \|s\|_{v}
$$

Hence, by the geometric-arithmetic mean inequality, we have

$$
\|s\|^{2}=\sum_{v \in S^{\infty}}\left\|s_{v}\right\|_{v}^{2} \geq n\left(\prod_{v \in S_{\infty}^{\infty}}\|s\|_{v}^{2}\right)^{1 / n} \geq n e^{-2 \widehat{\operatorname{deg}} \bar{E} /(r n)}=n N(\bar{E})^{-2 /(r n)} .
$$

This yields

$$
\lambda \geq \sqrt{n} N(\bar{E})^{-1 /(r n)} .
$$

Similarly, by Lemma 3.2,

$$
\lambda^{*} \geq \sqrt{n} N\left(\bar{E}^{*}\right)^{-1 /(r n)} .
$$


If $\widehat{\operatorname{deg}} \bar{E} \leq 0$, then $N(\bar{E}) \leq 1$ and hence $\lambda \geq \sqrt{n}$. By [G, Prop. 4.4], we obtain

$$
k^{0}(\bar{E}) \leq 1+\frac{3^{r n} \pi}{\pi-\log 3} e^{-\pi \lambda^{2}} .
$$

This implies (1).

Since $\gamma_{i} \leq r n$ for all $i$, Lemma 3.1 yields

$$
k^{0}(\bar{E}) \leq \omega^{r n} \max \left\{1,(r n / \lambda)^{r n}\right\} .
$$

If $\widehat{\operatorname{deg}} \bar{E} \geq 0$, then $\max \left\{1,(r n / \lambda)^{r n}\right\} \leq(r \sqrt{n})^{r n} e^{\widehat{\operatorname{deg}} \bar{E}}$. Thus (2) follows.

To prove (3), we note that, by Lemma 3.1 we have either

$$
k^{0}(\bar{E}) \leq \omega^{r n} \max \{1,1 / \lambda\}^{r n / 2}(r n)^{r n}
$$

or

$$
k^{0}\left(\bar{E}^{*}\right) \leq \omega^{r n} \max \left\{1,1 / \lambda^{*}\right\}^{r n / 2}(r n)^{r n} .
$$

By the assumption $0 \leq \widehat{\mu}(\bar{E}) \leq \widehat{\operatorname{deg}} \bar{\omega}_{S}$, we have $\widehat{\operatorname{deg}} \bar{E} \geq 0$ and $\widehat{\operatorname{deg}} \bar{E}^{*} \geq 0$. Assume that $(*)$ holds. Since $\max \{1,1 / \lambda\}=1 / \lambda$, we have

$$
h^{0}(\bar{E}) \leq r n(\log \omega+\log r n)+\widehat{\operatorname{deg}} \bar{E} / 2 .
$$

Hence we are done in this case. Similarly, if $(* *)$ holds, we obtain

$$
h^{0}\left(\bar{E}^{*}\right) \leq r n(\log \omega+\log r n)+\widehat{\operatorname{deg}} \bar{E}^{*} / 2,
$$

which yields, by Riemann-Roch,

$$
h^{0}(\bar{E}) \leq r n(\log \omega+\log r n)+\widehat{\operatorname{deg}} \bar{E} / 2 .
$$

This completes the proof.

4. An example. In this section we shall give an example of semistable Hermitian bundle due to J.-B. Bost. For higher-dimensional Arakelov geometry, we refer to $[\mathrm{SABK}]$.

Let $A$ be an abelian variety of dimension $g$ over a number field $K$ with $n=[K: \mathbb{Q}]$. Let $L$ be an ample symmetric line bundle on $A$ and let $\chi(A, L)$ denote its Euler characteristic. Then, by Riemann-Roch,

$$
\chi(A, L)=L^{g} / g !
$$

Assume that $A$ has good reduction and let the abelian scheme

$$
\pi: \mathcal{A} \rightarrow S=\operatorname{Spec} \mathcal{O}_{K}
$$

denote a model of $A$ over $S$ and let $\mathcal{L}$ be a line bundle $\mathcal{A}$ extending $L$. Let $\varepsilon: S \rightarrow \mathcal{A}$ be a zero section. For each $v \in S^{\infty}$ there exists an $F_{\infty^{-}}$ invariant Hermitian metric \|\|$_{v}$ on $L_{v}$ such that its curvature form is translation invariant. This metric is unique up to multiplication by positive constants. Let $\overline{\mathcal{L}}$ denote the Hermitian line bundle equipped with this metric, normalized so that $\varepsilon^{*} \overline{\mathcal{L}}$ is isometric to the trivial line bundle $\overline{\mathcal{O}}_{S}$ with 
the trivial metric. Then $\pi_{*} \mathcal{L}$ is a vector bundle of rank $\chi(A, L)$ on $S$. We equip it with the metric which is defined as follows. For each $v \in S^{\infty}$ and $s \in\left(\pi_{*} \mathcal{L}\right)_{v} \cong H^{0}\left(A_{v}(\mathbb{C}), \mathcal{L}_{v}\right)$, one sets

$$
\|s\|_{v}=\int_{\mathcal{A}_{v}(\mathbb{C})}\|s\|_{\mathcal{L}}^{2} d \mu
$$

where $d \mu$ denotes the Haar measure of total volume one on $\mathcal{A}_{v}(\mathbb{C})$. J.-B. Bost proved that the resulting Hermitian bundle $\pi_{*} \overline{\mathcal{L}}$ is semistable and its slope is given by

$$
\widehat{\mu}\left(\pi_{*} \overline{\mathcal{L}}\right)=-\frac{1}{2} h(A)+\frac{1}{4} \log \left(\frac{\chi(A, L)}{(2 \pi)^{g}}\right),
$$

where $h(A)$ denotes the Faltings height of $A$ ([B,Théorème 4.2]). Thus, we may apply Theorem 2.2 to $\pi_{*} \overline{\mathcal{L}}$ to see that if

$$
\log \left(\frac{\chi(A, L)}{(2 \pi)^{g}}\right) \geq 2 h(A)
$$

then

$$
\begin{aligned}
h^{0}\left(\pi_{*} \overline{\mathcal{L}}\right) \leq & \chi(A, L)\left\{n\left(\log \omega+\frac{1}{2} \log n+\log \chi(A, L)\right)\right. \\
& \left.-\frac{1}{2} h(A)+\frac{1}{4} \log \left(\frac{\chi(A, L)}{(2 \pi)^{g}}\right)\right\} .
\end{aligned}
$$

\section{References}

[B] J.-B. Bost, Périodes et isogénies des variétés abéliennes sur les corps de nombres (d'après D. Masser et G. Wüstholz), Astérisque 237 (1996), 115-161.

[BGN] L. Brambila-Paz, I. Grzegorczyk and P. E. Newstead, Geography of BrillNoether loci for small slopes, J. Algebraic Geom. 6 (1997), 645-669.

[GS] G. van der Geer and R. Schoof, Effectivity of Arakelov divisors and the theta divisor of a number field, Selecta Math. (N.S.) 6 (2000), 377-398.

[G] R. P. Groenewegen, An arithmetic analogue of Clifford's theorem, J. Théor. Nombres Bordeaux 13 (2001), 143-156.

[SABK] C. Soulé, D. Abramovich, J.-F. Burnol and J. Kramer, Lectures on Arakelov Geometry, Cambridge Univ. Press, 1992.

Department of Mathematics

Tokyo Metropolitan University

Minami-Ohsawa 1-1, Hachioji-shi

Tokyo 192-0397, Japan

E-mail: nakasima@comp.metro-u.ac.jp 